\title{
The CMS tracking algorithms and their performance in cosmic ray data
}

\author{
Antonio Tropiano*† \\ Università di Firenze and INFN \\ E-mail: tropianoefi.infn.it
}

The CMS tracker is the largest silicon detector ever constructed, with about 200 square meters of active area and over 70 million readout channels. The tracker will operate in a very difficult environment, due to the large track multiplicity that will arise from LHC collisions. The amount of material the tracker itself is made of will make the task of track reconstruction at CMS even more challenging. During the 2008 Cosmic Run at Four Tesla (CRAFT) the CMS tracker operated for the first time with the rest of the CMS detectors and about 6 millions cosmic muons were reconstructed. In 2009 the data taking was repeated and this time about 12 millions cosmic muons were reconstructed. With this amount of data it was possible to test the detector performances, to align the detector and to test the CMS track reconstruction algorithms with real cosmic events. I describe the main algorithms used for track reconstruction at CMS and I show the results achieved with the cosmic ray data collected by CMS during CRAFT08 and CRAFT09.

9th International Conference on Large Scale Applications and Radiation Hardness of Semiconductor Detectors

30 September - 2 October 2009

Florence, Italy

\footnotetext{
*Speaker.

$\dagger$ on behalf of the CMS collaboration
} 


\section{The CMS tracker detector}

The CMS silicon tracker [1] is shown in figure 1. It is the largest silicon detector ever constructed. It is $5.4 \mathrm{~m}$ long and its radius is about $1.2 \mathrm{~m}$. It is made of pixel layers, in the innermost part, and strip layers, in the outermost. Pixels and strips are arranged into cylindrical layers in the barrel part, named Pixel Barrel, Tracker Inner Barrel (TIB) and Tracker Outer Barrel (TOB). The disks placed in the endcap regions, named Pixel EndCap, Tracker Inner Disk (TID) and Tracker EndCap (TEC) allow the coverage of a great portion of solid angle. With this design the CMS tracker covers the $\eta$ region between -2.5 and 2.5.

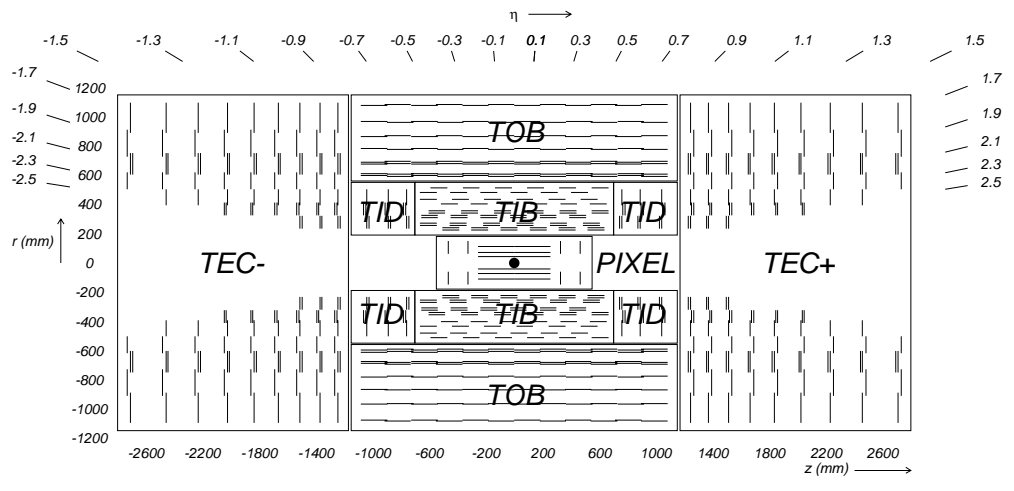

Figure 1: A cross section through the CMS tracker. The lines represents the modules. Double lines indicates stereo modules, which give a two dimensional measurement of the point of passage of the particle.

\section{The CMS tracking algorithms}

The track reconstruction in CMS is divided into four steps. The first step is the local reconstruction. In this step the signals that comes from the strips and pixels are turned into hits, which represents the measured point of passage of the particle and the associated error.

In the second step, the seed finding is performed. It consists in estimating the parameters of the tracks, using a subset of the total hits reconstructed in the tracker. They can be collected in the inner layers of the tracker or in the outer, depending from the algorithm used.

The third step is the pattern recognition. In this step hits are added to the tracks reconstructed in the seeding step, using the information of all other hits reconstructed.

In the fourth step the final fit is performed. Hits collected during the pattern recognition are used to give the final estimate the tracks parameters.

The main tracking algorithm implemented in CMS [2] is the Combinatorial Track Finder (CTF). In this algorithm the seeding step is done using the innermost layers of the tracker (pixels and first layers of TIB). The pattern recognition step is based on the Kalman Filter [3]. The trajectory parameters are propagated and updated from the innermost to the outermost layers. The hits to assign to the track are searched in a narrowing window as the track approaches external layers. The final fit is done running two Kalman Filter in the opposite directions (one inside-out 
and one outside-in).

There are also other tracking algorithms implemented in CMS, devoted to the reconstruction of electrons (Gaussian Sum Filter) or to the reconstruction of high density jets (Deterministic Annealing Filter) but all the results in this paper refers to the CMS standard reconstruction algorithm, the iterative tracking.

\subsection{The iterative tracking}

The CMS standard reconstruction algorithm is the iterative tracking, which is essentially an iterated CTF. The track reconstruction is repeated several times, each time with different settings. In the current version of the software it is made of 6 steps.

The first steps are devoted to the reconstruction of particles from the primary interaction vertex. In these steps the seeding is done using the innermost layers of the tracker. The last steps are instead devoted to the reconstruction of tracks detached from the primary vertex; for this reason the seeding is done using hits from the outermost tracker layers.

The iterative tracking steps are divided as in the following. There is an initial fit of the tracks using the CTF algorithm. Then the hits already associated to a track are removed. This allows to perform a second fit in a less crowded environment, using only hits that were not previously assigned to a track.

\section{The tracking performance in collisions}

In figure 2 the reconstruction efficiency and fake rate of the CMS standard tracking algorithm are shown for different types of simulated events. The reconstruction efficiency for muons is almost $100 \%$ for all the $\eta$ range, but there is a slight degradation of tracking performances for pions, electrons and multitrack events as $t \bar{t}$ production, especially in the transition region between barrel and endcap. This can be explained by the large amount of material in this region, that causes pions to inelastically interact with the material. After the interaction pions are lost and so can leave too few hits in the tracker to be correctly reconstructed. Electrons lose energy via bremsstrahlung radiation and for this reason the pattern recognition can fail.

In figure 3 the track impact parameters resolution for muon reconstruction is shown. The resolution on the transverse impact parameter $d_{0}$ varies between $10 \mu \mathrm{m}$ for $100 \mathrm{GeV} / \mathrm{c}$ muons and $50-60 \mu \mathrm{m}$ for $1 \mathrm{GeV} / \mathrm{c}$ muons (in the barrel region).

The $p_{T}$ resolution is shown in figure 4 . It is less than $1 \%$ for tracks with $p_{T}$ from 1 to $10 \mathrm{GeV} / \mathrm{c}$, in the barrel region and less than $2 \%$ for tracks with a $p_{T}$ of $100 \mathrm{GeV} / \mathrm{c}$. The resolution decreases in the forward region due to the smaller lever arm.

\section{The CMS tracking algorithms for cosmic-ray events}

The CMS tracking algorithms have also been adapted to deal with cosmic-ray events. Currently there are two tracking algorithms dedicated to cosmic rays reconstruction, the CTF for cosmic-rays and the Cosmic Track Finder. 


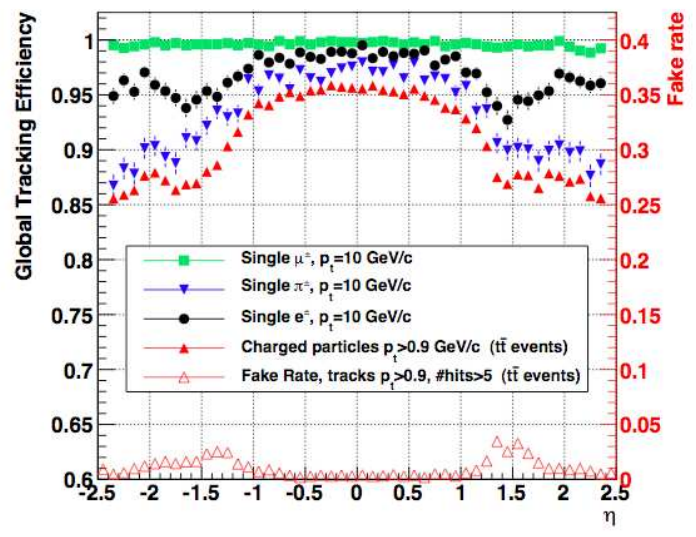

Figure 2: Efficiency and fake rate of the standard CMS tracking algorithm for different kinds of simulated events.
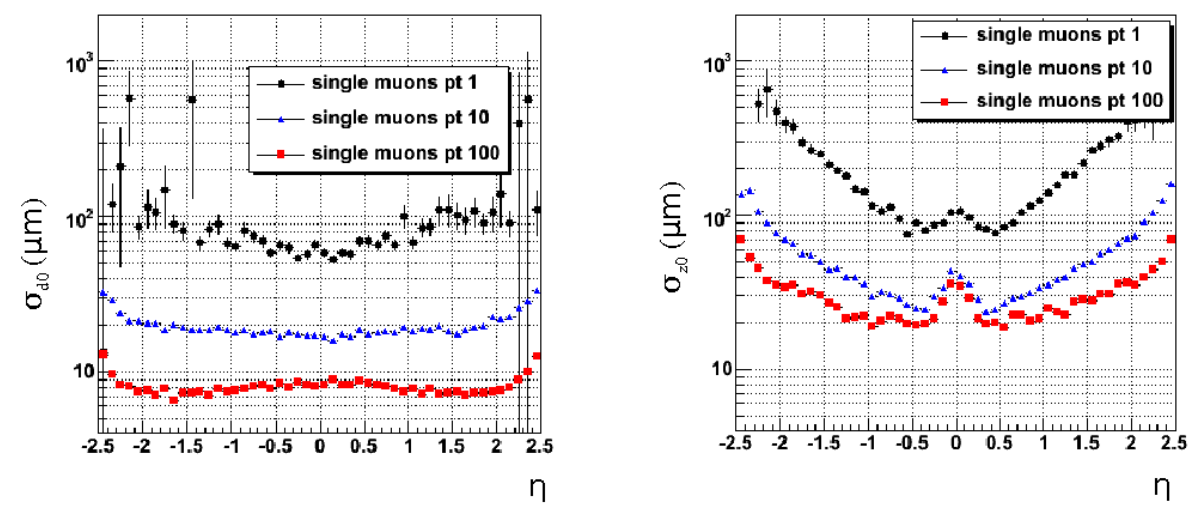

Figure 3: Resolution of the tranverse impact parameter $d_{0}$ and longitudinal impact parameter $d_{z}$ (or $z_{0}$ ) as a function of $\eta$, for muons with a $p_{T}$ of 1,10 and $100 \mathrm{GeV} / \mathrm{c}$.

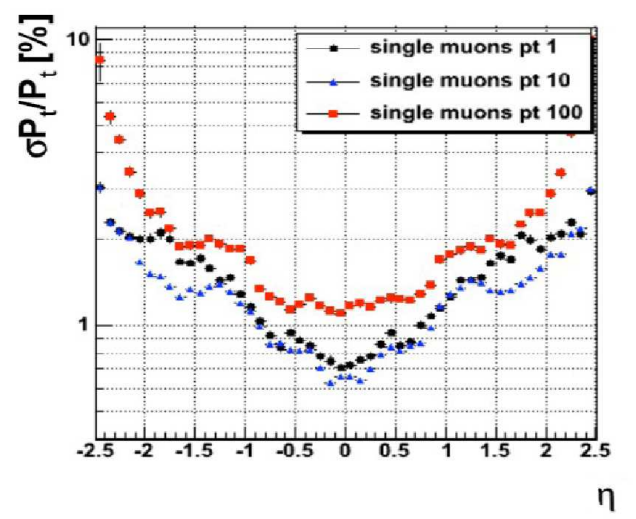

Figure 4: Resolution of the transverse momentum $p_{T}$ as a function of $\eta$, for muons with a $p_{T}$ of 1,10 and $100 \mathrm{GeV} / \mathrm{c}$. 
The CTF for cosmic is the standard CTF with a modified seeding step. In fact, since cosmic rays enter the apparatus from the outside, moving inwaard from the top, contrary to the collision case, the seeding is done using TOB and TEC layers. The pattern recognition step and the final fit are the same as for collisions.

The Cosmic Track Finder is an algorithm dedicated to cosmic reconstruction only. The seeding is done using hits in TIB or in TOB. Then, in the pattern recognition step, hits are aligned from the top to the bottom and a propagation based on a Kalman Filter is performed. The final fit is done running a Kalman Filter in the direction opposite to the pattern recognition one. At the end of the process only one track per event is retained.

\section{The tracking performance in cosmics}

During the period between October and November 2008 the whole CMS tracker operated together with the other subdetectors, with the B field at 3.8 Tesla. This exercise has been named CRAFT (Cosmic Run At Four Tesla). It was the first operation of the tracker together with all CMS subdetectors. Cosmic rays were triggered by the muon chambers and were used to test the performance of the CMS tracking system. Data collected with CRAFT 08 were successfully used to calibrate and test the performance of the CMS tracker. This exercise has been repeated in JulySeptember 2009 (CRAFT 09).

During the 2008 and 2009 data taking the number of collected tracks with hits in the tracker have been several millions, providing a large amount of data to test the performance of the CMS tracker and of CMS tracking algorithms. In table 1 the percentage of operational modules in pixel and strips both in CRAFT 08 and CRAFT 09 data taking. We can see that the percentage of operational modules is close to $100 \%$ for both periods of data taking.

\begin{tabular}{|c|c|c|}
\hline Tracker region & CRAFT 08 & CRAFT 09 \\
\hline TOB & $98 \%$ & $98.3 \%$ \\
\hline TIB/TID & $96.6 \%$ & $96.5 \%$ \\
\hline TEC+ & $99.2 \%$ & $98.8 \%$ \\
\hline TEC- & $97.8 \%$ & $99.2 \%$ \\
\hline Pixel Barrel & $99.1 \%$ & $99.1 \%$ \\
\hline Pixel Endcap & $94.0 \%$ & $96.9 \%$ \\
\hline
\end{tabular}

Table 1: Percentage of operating modules for CRAFT 08 and CRAFT 09.

\subsection{Measurement of tracking efficiency with cosmics}

With this large amount of tracks it was possible to measure the tracking algorithm efficiency using real data. Three methods have been developed to measure tracking efficiency.

The "tag and probe" method is based on the matching of a muon reconstructed in the muon chambers with one reconstructed in the tracker. The muon is reconstructed in the muon chambers combining hits coming from the top and the bottom hemispheres. The track is then propagated in the tracker volume and is matched to a track reconstructed using only the tracker information. The 
efficiency is estimated with respect to muons satisfying topological requirements similar to those typical of muons produced by collisions, applying several cinematic cuts, requiring for example that the point of closest approach of the extrapolated muon to the centre of the detector is less than $30 \mathrm{~cm}$ in both the transverse and longitudinal directions. Table 2 shows the efficiency measured with this method for both cosmic CTF and Cosmic Track Finder in CRAFT 08 data, while figure 5 shows the same efficiency, but obtained with CRAFT 09 data. Reconstruction efficiencies are close to $100 \%$ for both reconstruction algorithms and in good agreement with Monte Carlo predictions.

\begin{tabular}{|c|c|c|}
\hline Algorithm & Data efficiency & Monte Carlo efficiency \\
\hline CTF & $(99.78 \pm 0.02) \%$ & $(99.88 \pm 0.00) \%$ \\
\hline Cosmic Track Finder & $(99.47 \pm 0.04) \%$ & $(99.72 \pm 0.01) \%$ \\
\hline
\end{tabular}

Table 2: Efficiency evaluated with the tag and probe method, for CRAFT 08 data.

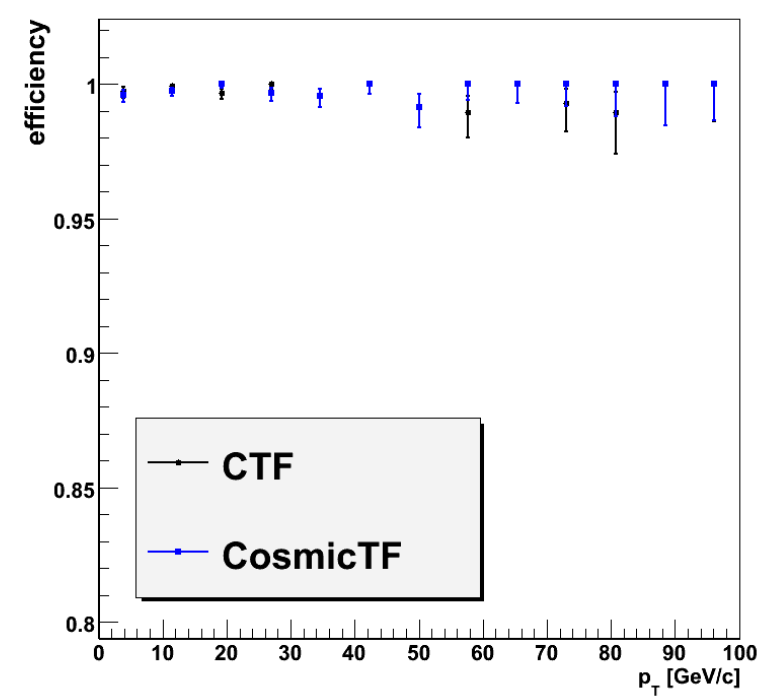

Figure 5: Efficiency as a function of $p_{T}$, evaluated with the tag and probe method, for CRAFT 09 data.

The second method used to estimate the tracking efficiency is the so-called "split tracks method". The tracker is divided in an upper and a lower hemisphere. Then tracks are reconstructed indipendently in both sides of the detector. Finally the track reconstructed in the top half is matched to the one reconstructed in the bottom half and viceversa. With this method two efficiencies can be computed. Even in this case the reconstruction efficiency is evaluated with respect to muons satisfying topological requirements similar to those typical of muons produced by collisions and the same cuts as in the previous method are applied.

In figure 6 is shown the tracker efficiency measured with this method, for CRAFT 08 data. In the figure both efficiencies of finding a track in the top half given a track in the bottom, $\varepsilon(T \mid B)$, and viceversa, $\varepsilon(B \mid T)$, are shown. Even in this case the efficiency is close to $100 \%$ and in good agreement with Monte Carlo predictions. The observed difference between $\varepsilon(T \mid B)$ and $\varepsilon(B \mid T)$ is due to some TOB inactive regions used for seeding the tracks. However these inactive regions don't affect 
the performance in collisions, since the seeding is done using the innermost layers of the tracker detector, and not TOB ones.

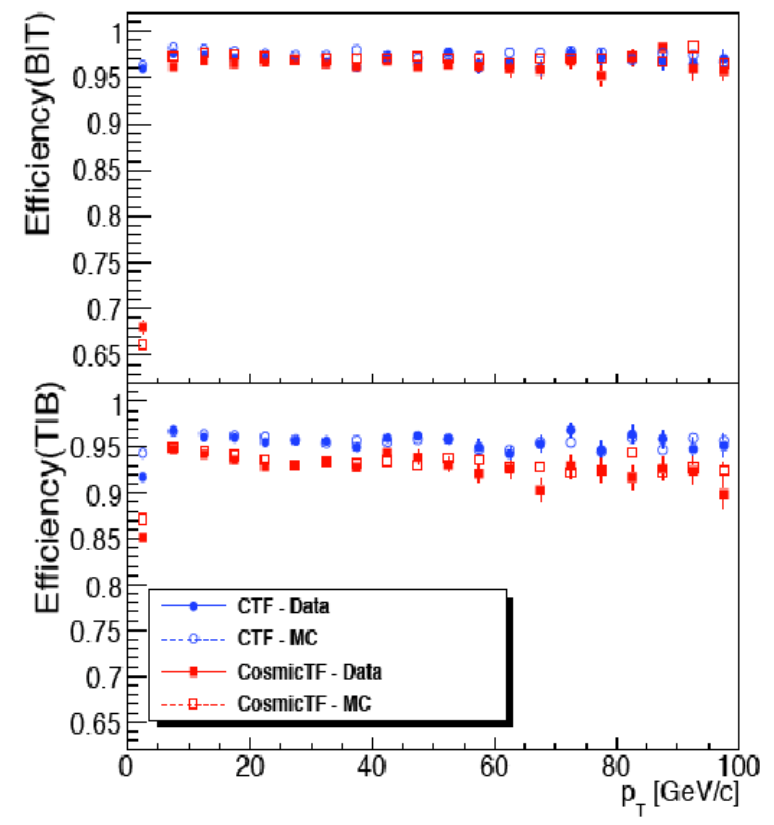

Figure 6: Efficiency as a function of $p_{T}$, evaluated with the split tracks method. Both $\varepsilon(T \mid B)$ and $\varepsilon(B \mid T)$ are shown.

A third method used to estimate the track reconstruction efficiency is the "collision-like" method. In this case tracks are reconstructed with settings similar to the ones used in reconstruction of tracks coming from collisions (i. e. fitting the trajectory from the innermost to the outermost layers). As in the second method two half tracks are reconstructed, but this time starting from the center of the detector. Then the efficiency is computed matching these tracks as in the split tracks method.

In table 3 the seed finding, pattern recognition and track reconstruction efficiencies, estimated using this method, for CRAFT 08 data, are shown. We can see that even with this method the track reconstruction efficiency is close to $100 \%$ and in agreement with Monte Carlo predictions.

\begin{tabular}{|c|c|c|}
\hline Efficiency & Data & Monte Carlo \\
\hline Seed finding & $(99.17 \pm 0.12) \%$ & $(99.30 \pm 0.08) \%$ \\
\hline Pattern recognition & $(99.79 \pm 0.06) \%$ & $(99.64 \pm 0.05) \%$ \\
\hline Track reconstruction & $(98.96 \pm 0.13) \%$ & $(98.94 \pm 0.09) \%$ \\
\hline
\end{tabular}

Table 3: Seed finding, pattern recognition and track reconstruction efficiency evaluated using the collisionlike method

\subsection{Measurement of track parameters resolution with cosmic-ray particles}

A measurement of the track parameters resolution has been done using CRAFT data. The method used to estimate the resolution is the following. Tracks fully reconstructed are split at the 
point of closest approach to the beamline. Then top and bottom segments are refitted indipendently. Each leg is finally propagated to its respective point of closest approach to the beamline and their parameters estimations at this point are compared. In figure 7 the results of this study are shown. The residuals obtained after the alignment show a good agreement with Monte Carlo predictions for a perfectly aligned scenario.
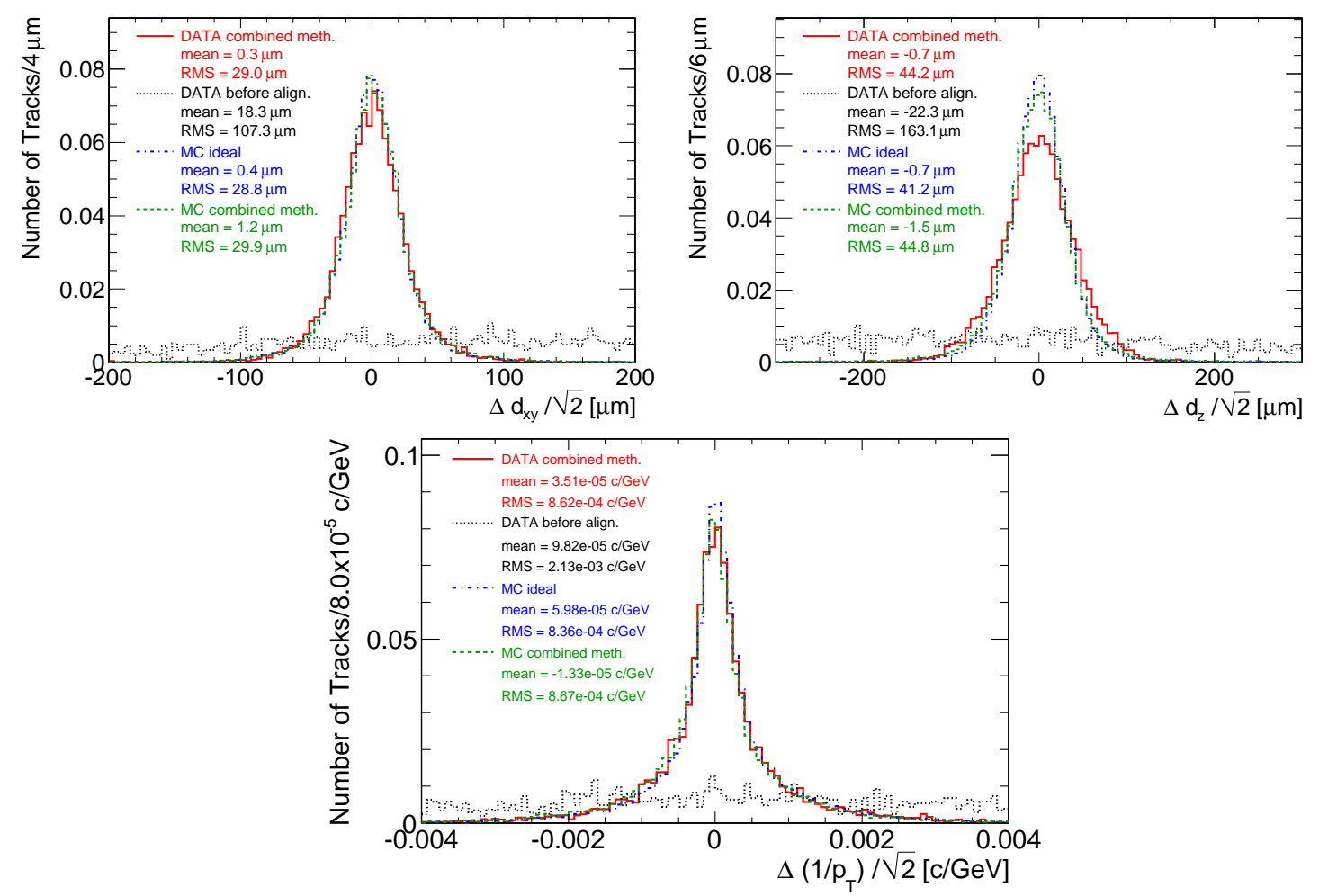

Figure 7: Normalized residuals of the transverse momentum, longitudinal and transverse impact parameters. They are shown for the data before alignment (in black), for the data after the alignment (in red), for the Monte Carlo in a perfectly aligned scenario (in blue) and for a Monte Carlo after the alignment (in green).

\section{Conclusions}

In conclusion we can say that, even if track reconstruction at CMS is a big challenge, the algorithms that CMS has developed have shown good performances. They have been tested on simulated events, with detector effects included in the simulation, and they have shown great performances for both reconstruction efficiency and tracks parameters estimation.

The CMS standard tracking algorithm (Combinatorial Track Finder) has also been modified to deal with the reconstruction of cosmic rays and has shown good performances during 2008 and 2009 cosmic data taking. Three different methods have been developed to estimate the track reconstruction efficiency in cosmic-ray events. They yield consistent results. Although results are similar, some small differences were observed. The main difference between the efficiencies determined by the first and second methods arises from the fact that tracks are sought in only one 
half of the detector in the second method, while in the first method, tracks may be found from seeds produced in both halves of the tracker. The measurements of the "Inside-out tracking method" give confidence that the track reconstruction will perform well in proton-proton collisions. Finally, the efficiencies measured in the Monte Carlo simulation agree very well with those measured in the data once the known detector inefficiencies are accounted for in the simulation. This indicates that the tracker and the reconstruction algorithms are well understood.

The full potential of the CMS tracker will be clear when collision will happen and CMS tracking algorithms will show their potential in the very crowded environment created by proton collisions at the LHC.

\section{References}

[1] CMS Collaboration, The CMS experiment at the CERN LHC, JINST 3 S08004 (2008)

[2] W. Adam et al., Track Reconstruction in the CMS tracker, CMS Note 2006/041 (2005).

[3] R. Frühwirth, Application of Kalman filtering to track and vertex fitting, Nucl. Instrum. Meth., A 262, (1987). 